\title{
Estimativa de carbonato de cálcio aplicado via água de irrigação nas regiões da Chapada do Apodi e Baixo Açu, RN
}

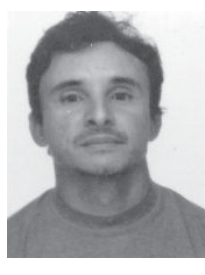

Celsemy E. Maia' ${ }^{1}$ Elis R.C. de Morais² \& Maurício de Oliveira ${ }^{3}$

\author{
1 ESAM. R. Miro F. de Mendonça, 130, Planalto 13 de Maio, CEP 59633-010, Mossoró, RN. E-mail: celsemymaia@bol.com.br (Foto) \\ 2 AGROMASTER \\ ${ }^{3}$ ESAM. E-mail: mauricio@esam.br
}

Protocolo $086-11 / 07 / 2000$

\begin{abstract}
Resumo: A aplicação de água com elevados teores de carbonato e bicarbonato pela irrigação, pode contribuir para o aumento do $\mathrm{pH}$ dos solos após alguns anos de cultivo. Esse trabalho teve como objetivo avaliar a água de irrigação quanto a seu teor de carbonato e bicarbonato, baseado no conceito do equivalente carbonato de cálcio $\left(\mathrm{E}_{\mathrm{CaCO}_{3}}\right)$ da água de irrigação nas regiões da Chapada do Apodi e Baixo Açu no Estado do Rio Grande do Norte. Considerando uma lâmina de irrigação de $400 \mathrm{~mm}$, os resultados mostraram que as águas da região da Chapada do Apodi apresentaram maiores valores de $\mathrm{E}_{\mathrm{CaCO}_{3}}$ quando comparadas com as água da região do Baixo Açu. Na região da Chapada do Apodi os maiores valores de $\mathrm{E}_{\mathrm{CaCO}_{3}}$ independente da origem foram para as águas da região de Mossoró, com média de $765 \mathrm{~kg} \mathrm{ha}^{-1}$ e o menor valor foi para a região de Grossos e Upanema com $626 \mathrm{~kg} \mathrm{ha}^{-1}$. Para a região do Baixo Açu, verifica-se que, independente da origem das águas, a região de Ipanguassu apresentou os maiores valores de $\mathrm{E}_{\mathrm{CaCO}_{3}}$ com média de $654 \mathrm{~kg} \mathrm{ha}^{-1}$, e o menor valor foi para a região de Carnaubais com $580 \mathrm{~kg} \mathrm{ha}^{-1}$.
\end{abstract}

Palavras-chave: alcalinidade, precipitação de carbonato, deficiência nutricional, pH do solo

\section{Estimate of the calcium carbonate applied through irrigation water in Chapada do Apodi and Baixo Açu regions of Rio Grande do Norte, Brazil}

\begin{abstract}
The application of irrigation water with high contents of carbonate and bicarbonate can contribute to $\mathrm{pH}$ elevation of the soils after some years of cultivation. This study had as its objective the evaluation of the irrigation water with respect to its carbonate and bicarbonate content, based on the concept of the Equivalent Calcium Carbonate $\left(\mathrm{E}_{\mathrm{CaCO}_{3}}\right)$ in the region of the Chapada do Apodi and Baixo Açu in the state of Rio Grande do Norte, Brazil. Considering an irrigation water depth of $400 \mathrm{~mm}$, the results showed that the waters of the region of the Chapada do Apodi presented larger values of $\mathrm{E}_{\mathrm{CaCO}_{3}}$ compared to those of the region of Baixo Açu. In the region of the Chapada do Apodi, independent of the origin, largest values of $\mathrm{E}_{\mathrm{CaCO}_{3}}$ were found for the waters of the region of Mossoró, with an average of $765 \mathrm{~kg} \mathrm{ha}^{-1}$ and the smallest value was observed for Grossos and Upanema with $626 \mathrm{~kg} \mathrm{ha}^{-1}$. For the region of Baixo Açu, independent of the origin of the waters, the region of Ipanguassu presented the highest values of $\mathrm{E}_{\mathrm{CaCO}_{3}}$ with an average of $654 \mathrm{~kg} \mathrm{ha}^{-1}$ whereas the smallest values were found for the region of Carnaubais, with $580 \mathrm{~kg} \mathrm{ha}^{-1}$.
\end{abstract}

Key words: alkalinity, precipitation of carbonate, deficiency nutritional, soil pH

\section{INTRODUÇ̃̃̃O}

Para se prever um problema relacionado com a qualidade da água, tem que se avaliar seu potencial em criar condições no solo que possam restringir seu uso e avaliar a necessidade de empregar técnicas de manejos especiais para manter rendimentos aceitáveis das culturas. Existem vários procedimentos para realizar esta avaliação, porém, independente de método que se usa e, uma vez que estes problemas devem ser resolvidos à nível de parcela, a avaliação deve ser feita levando-se em conta as condições locais específicas e a capacidade de manejo do usuário (Ayers \& Westcot, 1991). Ainda segundo estes autores, o conceito de qualidade da água refere-se as suas características físico-químicas, que podem afetar a adaptabilidade para seu uso específico; em outras palavras, a relação entre a quantidade da água e as necessidades do usuário. A qualidade da água define-se por uma ou mais característica físicas, químicas ou biológicas. Usos específicos podem ter diferentes requisitos de qualidade. Assim, uma água pode ser considerada como de melhor 
qualidade, se produzir melhores resultados ou causar menos danos. No caso específico da agricultura irrigada, tem-se preparado numerosos guias para o uso de água segundo sua qualidade. Cada uma delas com certa utilidade, porém, nenhuma tem sido completamente satisfatória, devido principalmente, as variabilidades das condições de campo. Assim, dependendo da granulometria do solo, da sua mineralogia, e das relações catiônicas solo-solução, águas classificadas com diferentes graus de qualidade, podem exibir comportamento diferenciado nas alterações químicas do solo (Kelley, 1963; Inoue, 1984; Shainberg \& Letey, 1984).

Os principais íons analisados na água de irrigação são o cálcio, o magnésio, o potássio, o sódio, o cloreto, o sulfato, o carbonato e o bicarbonato. Com especial referência ao carbonato e bicarbonato, esses são os principais responsáveis pelo equilíbrio que governa o $\mathrm{pH}$ da água de irrigação. Os principais efeitos do excesso de carbonato e bicarbonato são os problemas de precipitação nas tubulações, diminuindo a eficiência de aplicação de água e fertilizantes quando se utiliza a irrigação localizada e se pratica a fertirrigação (Burt et al, 1995). Atualmente, verifica-se o contrário do que ocorre em outras regiões onde, áreas irrigadas com água com altos teores de carbonato e bicarbonato na região da Chapada do Apodi ocorre, um aumento nos valores de $\mathrm{pH}$ dos solos depois de alguns anos de cultivo. $\mathrm{O}$ aumento no $\mathrm{pH}$ do solo com os anos de cultivo é devido aos elevados teores de carbonato e bicarbonato na água de irrigação, principalmente as de poço tubular. Assim, a aplicação dessas águas ao solo tem um efeito no $\mathrm{pH}$ desses, que pode ser calculado em relação ao equivalente a carbonato de cálcio com poder relativo de neutralização total (PRNT) de $100 \%$.

Os solos da região da Chapada do Apodi são caracterizados por serem solos jovens, de boa fertilidade e, devido ser de origem calcária, apresentam $\mathrm{pH}$ natural de neutro a alcalino. Porém, com os cultivos sucessivos, em especial com a cultura do melão, verifica-se que com 3 anos de cultivo, o pH desses solos aumenta, ocorrendo o contrário do que ocorre em outras regiões, onde o $\mathrm{pH}$ dos solos decresce com os anos de cultivo, sendo necessário a aplicação de calcário para elevação do $\mathrm{pH}$ desses solos para uma faixa ideal para a cultura.

A região do Baixo Açu, situada no oeste do estado do Rio Grande do Norte, é um pólo hortifrutigranjeiro, onde são produzidos principalmente banana, tomate, melão e manga. A região possui uma extensa faixa de solo de origem calcária, porém nas margens dos rios formam-se as vázeas. A principal fonte de água da região é a barragem Armando Ribeiro Gonçalves, responsável pela irrigação na região. Ao contrário da região da Chapada do Apodi, as águas do Baixo Açu apresentam composição iônica menos salina, porém apresentam maior valor de carbonato de sódio residual e menor problema com relação a precipitação de carbonato e bicarbonato nas tubulações (Maia \& Morais, 1998). A água da região mesmo com teores de carbonato e bicarbonato inferiores aos verificados na Chapada do Apodi, tem mostrado que o uso contínuo dessa água está causando o aumento do $\mathrm{pH}$ dos solos após alguns anos de cultivo.

O objetivo desse trabalho foi avaliar a água de irrigação quanto a seu teor de carbonato e bicarbonato, baseado no conceito do Equivalente Carbonato de Cálcio da água de irrigação nas regiões da Chapada do Apodi e Baixo Açu no estado do Rio Grande do Norte.

\section{MATERIAL E MÉTODOS}

\section{Base de dados}

Os dados utilizados para a presente pesquisa foram provenientes dos boletins de análises de água emitidos pelo Laboratório de Análises de Água e Fertilidade do Solo da Escola Superior de Agricultura de Mossoró - RN (LAAFS/ESAM), entre os anos de 1990-1995, num total de 607 amostras de águas de diferentes mananciais. Os dados foram cadastrados através do software SCASA (Maia \& Morais, 1996) e avaliados pelo software ALKA (Egreja Filho et al., 1999).

\section{Fisiografia da área de abrangência do estudo}

A microrregião Salineira Norte-Rio-Grandense é atualmente composta por 8 municípios (Alto do Rodrigues, Areia Branca, Carnaubais, Grossos, Guamaré, Macau, Mossoró e Pendências) enquanto a microrregião Açu e Apodi é constituída de 15 municípios (Assu, Apodi, Campo Grande, Caraúbas, Felipe Guerra, Governador Dix-Sept Rosado, Ipanguassu, Itajá, Itau, Janduís, Paraú, São Rafael, Serra do Mel, Severiano Melo e Upanema). Comercialmente, no entanto, as regiões de maior expressividade agrícola são denominadas de Baixo Açu (que compreende a região e enclaves da planície aluvional do baixo curso do Rio Piranhas-Açu) - liderada pela cidade do Assu e Chapada do Apodí - liderada comercialmente pela cidade de Mossoró.

As áreas supra mencionadas estão localizadas no extremo noroeste do Estado do Rio Grande do Norte, na quadrícula geográfica entre os Paralelos $4^{\circ} 48^{\prime}$ a $5^{\circ} 41^{\prime}$ 'de latitude Sul e os Meridianos 37030' a $38^{\circ} 5^{\prime}$ a Oeste de Greenwich. Nas áreas estudadas predomina o tipo bioclimático de Gausen $4 \mathrm{aTh}$ - Tropical quente de seca acentuada, com índice xerotérmico entre 150 e 200. Pela classificação de Thornthwaite que está baseada numa série de índices térmicos utilizando-se o balanço hídrico, as regiões apresentam clima do tipo DdA'a', ou seja, semi-árido megatérmico com pouco ou nenhum excesso de água durante o ano. Pelo regime térmico e pluviométrico, as regiões apresentam um clima do tipo BSw'h' pela classificação de Köppen. As chuvas têm distribuição bastante irregular no tempo e no espaço, aumentando sobremaneira o risco climático. A média anual de precipitação é de aproximadamente $679 \mathrm{~mm}$. Devido a baixa latitude e a ausência de fatores geográficos influenciadores, a temperatura apresenta-se sem grande variação anual. A média anual de temperatura é de aproximadamente $27,5^{\circ} \mathrm{C}$, sendo que o mês mais quente é dezembro, com média de $28,5^{\circ} \mathrm{C}$, e o mês mais frio é julho, com média de $26,5^{\circ} \mathrm{C}$. As temperaturas máximas e mínimas do ar têm valores médios iguais a 33,3 e $22,7^{\circ} \mathrm{C}$ respectivamente (Carmo Filho et al, 1991).

\section{Análise de água}

Nas análises de água realizadas pelo Laboratório de Análise de Água e Solos da ESAM, foram determinadas as seguintes características físico-químicas: $\mathrm{pH}, \mathrm{CE}$, cátions $\left(\mathrm{Ca}^{2+}, \mathrm{Mg}^{2+}, \mathrm{Na}^{+}\right.$ e $\left.\mathrm{K}^{+}\right)$e ânions $\left(\mathrm{Cl}^{-}, \mathrm{HCO}_{3}^{-} \mathrm{e} \mathrm{CO}_{3}^{2-}\right)$. É utilizada para determinação dessas características a metodologia proposta por Richards (1954). 
Equivalente carbonato de cálcio ( $\left.\mathrm{E}_{\mathrm{CaCO}_{3}}\right)$

Quando se aplica calcário ao solo, 1,0 mol de $\mathrm{H}^{+}$é neutralizado por $0,5 \mathrm{~mol} \mathrm{de} \mathrm{CaCO}_{3}$, de acordo com o equilíbrio (1)

$$
\mathrm{CaCO}_{3}+2 \mathrm{H}^{+} \Leftrightarrow \mathrm{Ca}^{2+}+\mathrm{CO}_{2}+\mathrm{H}_{2} \mathrm{O}
$$

O equilíbrio (1) resume 2 reações:

$$
\begin{aligned}
& \mathrm{CaCO}_{3}+\mathrm{H}^{+} \Leftrightarrow \mathrm{Ca}^{2+}+\mathrm{HCO}_{3}{ }^{-} \\
& \mathrm{HCO}_{3}{ }^{-}+\mathrm{H}^{+} \Leftrightarrow \mathrm{Ca}^{2+}+\mathrm{H}_{2} \mathrm{CO}_{3}{ }^{\circ}
\end{aligned}
$$

Desse modo, pode-se afirmar que $1,0 \mathrm{~mol} \mathrm{de} \mathrm{CO}_{3}{ }^{2-}$ neutraliza 2,0 mol de $\mathrm{H}^{+}$e 1,0 mol de $\mathrm{HCO}_{3}^{-}$neutraliza $1,0 \mathrm{~mol} \mathrm{de} \mathrm{H}^{+}$. Utilizando o conceito da equivalência química pode-se afirmar que:

$$
1,0 \mathrm{mmol}_{\mathrm{c}} \mathrm{CO}_{3}^{2-}=1,0 \mathrm{mmol}_{\mathrm{c}} \mathrm{HCO}_{3}^{-}=1,0 \mathrm{mmol}_{\mathrm{c}} \mathrm{CaCO}_{3}
$$

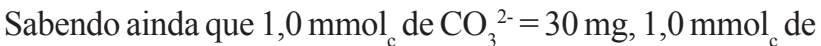
$\mathrm{HCO}_{3}^{-}=61 \mathrm{mg}$ e $1,0 \mathrm{mmol}_{\mathrm{c}} \mathrm{de}^{\mathrm{c}} \mathrm{CaCO}_{3}=50 \mathrm{mg}$ e fazendo os cálculos em separado para o carbonato e bicarbonato, tem-se:

$$
\mathrm{E}_{\mathrm{CaCO}_{3}}=\left(\mathrm{A}_{\mathrm{CO}_{3}{ }^{-}}+\mathrm{A}_{\mathrm{HCO}_{3}}{ }^{-}\right) * 50
$$

onde $\mathrm{A}_{\mathrm{CO}_{3}}$ e $\mathrm{A}_{\mathrm{HCO}_{3}}$ representam, respectivamente concentração de carbonatos e bicarbonatos na água.

Como os resultados de carbonato e bicarbonato são dados em mmol $\mathrm{L}^{-1}$ o resultado do $\mathrm{E}_{\mathrm{CaCO}_{3}}$ é em mg L $\mathrm{L}^{-1}$. Considerando que na irrigação as lâminas são aplicadas em $\mathrm{mm}$, e que $1 \mathrm{~mm}$ equivale a $1,0 \mathrm{~L} \mathrm{~m}^{-2}$, ou seja, $10.000 \mathrm{~L} \mathrm{ha}^{-1}$, tem-se:

$$
\begin{gathered}
\mathrm{ECaCO}_{3}=\frac{50 *\left(\mathrm{ACO}_{3}+\mathrm{AHCO}_{3}\right) * \mathrm{~h} * 10.000}{1.000 .000} \\
\mathrm{ECaCO}_{3}=\frac{\mathrm{ACO}_{3}+\mathrm{AHCO}_{3}}{2} * \mathrm{~h}
\end{gathered}
$$

onde:

$\mathrm{E}_{\mathrm{CaCO}_{3}}$ - equivalente carbonato de cálcio PRNT 100\% $\left(\mathrm{kg} \mathrm{ha}^{-1}\right)$

$\mathrm{A}_{\mathrm{CO}_{3}}$ - teor de carbonato na água de irrigação $\left(\mathrm{mmol}_{\mathrm{c}} \mathrm{L}^{-1}\right)$

$\mathrm{A}_{\mathrm{HCO}_{3}}$ - teor de bicarbonato na água de irrigação $\left(\mathrm{mmol}_{\mathrm{c}} \mathrm{L}^{-1}\right)$

h ${ }^{3}$ - lâmina de água aplicada (mm).

\section{RESULTADOS E DISCUSSÃO}

Os dados do Tabela 1 mostram que, independente da origem das águas, a região de Mossoró apresentou os maiores valores do Equivalente Carbonato de Cálcio $\left(\mathrm{E}_{\mathrm{CaCO}_{3}}\right)$ com média de $765 \mathrm{~kg} \mathrm{ha}^{-1}$ e o menor valor foi para a região de Grossos e Upanema com $626 \mathrm{~kg} \mathrm{ha}^{-1}$.

Levando em consideração a origem da água, os maiores valores de $\mathrm{E}_{\mathrm{CaCO}_{3}}$ foram para as água de rio da região de Upanema com $\mathrm{E}_{\mathrm{CaCO}_{3}}=920 \mathrm{~kg} \mathrm{ha}^{-1}$, e os menores valores para as águas de rio da região de Mossoró, com média de $630 \mathrm{~kg} \mathrm{ha}^{-1}$.

Esses dados mostram que, mesmo observando regiões com maiores valores de $\mathrm{E}_{\mathrm{CaCO}_{3}}$, todos os valores são elevados. Levando
Tabela 1. Equivalente carbonato de cálcio $\left(\mathrm{E}_{\mathrm{CaCO}_{3}}\right)$ para algumas cidades da região da Chapada do Apodi

\begin{tabular}{lcc} 
Cidade/Origem & $\begin{array}{c}\mathrm{CO}_{3}{ }^{2-}+\mathrm{HCO}_{3}{ }^{-} \\
\mathrm{mmol}_{\mathrm{c}} \mathrm{L}^{-1}\end{array}$ & $\begin{array}{c}\mathrm{E}_{\mathrm{CaCO} 3}{ }^{*} \\
\left(\mathrm{~kg} \mathrm{ha}^{-1}\right)^{*}\end{array}$ \\
\hline Apodi & 2,93 & 671 \\
Poço tubular & 2,75 & 644 \\
Poço amazonas & 2,79 & 651 \\
Baraúna & 3,07 & 706 \\
Poço tubular & 3,10 & 714 \\
Poço amazonas & 3,29 & 790 \\
Governador Dix-Sept Rosado & 2,98 & 680 \\
Poço tubular & 2,95 & 684 \\
Poço amazonas & 3,15 & 732 \\
Rio & 2,75 & 636 \\
Grossos & 2,69 & 626 \\
Poço tubular & 3,02 & 699 \\
Poço amazonas & 2,72 & 654 \\
Mossoró & 3,37 & 765 \\
Poço tubular & 3,26 & 742 \\
Poço amazonas & 3,01 & 698 \\
Rio & 2,72 & 630 \\
Caraúbas & 2,72 & 654 \\
Poço tubular & 3,29 & 790 \\
Poço amazonas & 3,29 & 790 \\
Upanema & 2,69 & 626 \\
Poço tubular & 2,75 & 644 \\
Poço amazonas & 3,15 & 732 \\
Rio & 3,74 & 920 \\
\hline Os & & \\
\hline
\end{tabular}

Os Valores de $\mathrm{CO}_{3}^{2-}+\mathrm{HCO}_{3}^{-}$e $\mathrm{E}_{\mathrm{CaCO}}$ são valores médios

(") Para uma lâmina de $400 \mathrm{~mm}$

em consideração uma lâmina de 400 mm, média de aplicação de água na cultura de melão por ciclo na região da Chapada do Apodi e, considerando 2 ciclos por ano, no caso seria aplicado aproximadamente $800 \mathrm{~mm}^{2} \mathrm{ano}^{-1}$, é de se esperar que esses solos apresentem, com o tempo, um aumento no $\mathrm{pH}$, pois equivaleria a aplicação de, em média, $1,4 \mathrm{Mg} \mathrm{ha}^{-1} \mathrm{ano}^{-1} \mathrm{de} \mathrm{CaCO}_{3}$ PRNT $100 \%$.

A principal implicação dessa quantidade de carbonato e bicarbonato aplicado no solo pode ser, por exemplo, uma precipitação de micronutrientes pelo aumento do $\mathrm{pH}$ do solo, bem como favorecendo a precipitação de fósforo na forma de fosfato de cálcio, já que, além do solo ser rico em cálcio, as águas também apresentam elevados teores do elemento. Isso sem contar que, a quantidade de carbonato e bicarbonato dessas águas pode precipitar dentro das tubulações e causar entupimento, prejudicando a uniformidade de distribuição da água e fertilizantes.

Observando a Tabela 2, verifica-se que, independente da origem das águas, a região de Ipanguassu apresentou os maiores valores

Tabela 2. Equivalente carbonato de cálcio $\left(\mathrm{E}_{\mathrm{CaCO}_{3}}\right)$ para algumas cidades da região do Baixo Açu

\begin{tabular}{lcc}
\hline Cidade/Origem & $\begin{array}{c}\mathrm{CO}_{3}{ }^{2-}+\mathrm{HCO}_{3}{ }^{-} \\
\mathrm{mmol}_{\mathrm{c}} \mathrm{L}^{-1}\end{array}$ & $\begin{array}{c}\mathrm{E}_{\mathrm{CaCO} 3} \\
\left(\mathrm{~kg} \mathrm{ha}^{-1}\right)^{*}\end{array}$ \\
\hline Assu & 2,75 & 644 \\
Poço tubular & 2,65 & 634 \\
Poço amazonas & 2,75 & 652 \\
Rio & 2,85 & 680 \\
Carnaubais & 2,38 & 580 \\
Poço amazonas & 2,38 & 594 \\
Ipanguassu & 2,83 & 654 \\
Poço amazonas & 2,92 & 678 \\
Rio & 2,72 & 654 \\
\hline
\end{tabular}

Os Valores de $\mathrm{CO}_{3}^{2-}+\mathrm{HCO}_{3}^{-}$e $\mathrm{E}_{\mathrm{CaCO}_{3}}$ são valores médios

(") Para uma lâmina de $400 \mathrm{~mm}$ 
de $\mathrm{E}_{\mathrm{CaCO}_{3}}$ com média de $654 \mathrm{~kg} \mathrm{ha}^{-1}$, e o menor valor foi para a região de Carnaubais com $580 \mathrm{~kg} \mathrm{ha}^{-1}$. Levando em consideração a origem da água, os maiores valores de $\mathrm{E}_{\mathrm{CaCO}_{3}}$ foram para a água de rio da região de Assu com $\mathrm{E}_{\mathrm{CaCO}_{3}}=680 \mathrm{~kg} \mathrm{ha}^{-1}$, e os menores valores para as águas de poço amazonas da região de Carnaubais, com média de $580 \mathrm{~kg} \mathrm{ha}^{-1}$.

Comparando esses dados com os obtidos para a região da Chapada do Apodi, verifica-se que, em média, os valores são 10 \% inferiores. Mesmo assim, para uma lâmina de $400 \mathrm{~mm}$ a quantidade $\mathrm{E}_{\mathrm{CaCO}_{3}}$ tem que ser levada em consideração. Isso porque, as águas dessa região apresentam teores de cálcio e magnésio inferiores aos verificados na região da Chapada do Apodi. Nesse caso, poderá haver um aumento do $\mathrm{pH}$ do solo sem o fornecimento desses dois nutrientes. $\mathrm{O}$ fato se torna mais complicado em decorrência do aumento relativo do sódio em relação ao cálcio e magnésio. Assim, pelo fato do carbonato e bicarbonato de sódio ser mais solúvel, a predominância do sódio no solo favorece o aumento do $\mathrm{pH}$ mais intensamente que na região da Chapada do Apodi. Atualmente já é visível o efeito desse mecanismo nos solos da região, principalmente no Perímetro Irrigado do Baixo Açu, que é intensificado ainda mais, pela baixa condutividade hidráulica da maioria dos solos do projeto.

Na região do Baixo Açu, há uma expansão no plantio da cultura da banana. Como a cultura é perene e pela alta evapotranspiração na região, aplica-se por ano aproximadamente 2.000 mm de água via irrigação. Assim, é aplicada via água uma grande quantidade de carbonato e bicarbonato que, traduzindo em $\mathrm{E}_{\mathrm{CaCO}_{3}}$, a tendência dos solos sob essa cultura é aumentar o $\mathrm{pH}$, principalmente naqueles solos sob condições de drenagem inadequadas.

Segundo Whipker et al. (1996), os teores de bicarbonatos e carbonatos são as principais formas químicas que contribui para a alcalinidade, mas hidróxidos dissolvidos, amônia, borato, bases orgânicas, fosfatos e silicatos também podem contribuem para a alcalinidade. Cita também que os íons carbonatos e bicarbonatos podem ter efeito tóxico para o crescimento das plantas. Esse efeito tóxico acredita-se ser devido a interferência na absorção pelas raízes de elementos essenciais e associado ao aumento no pH na solução do solo, do que a absorção direta do íons carbonatos e bicarbonato pelas plantas, além disso águas com excessiva alcalinidade também reduz a disponibilidade de micronutriente para as plantas pelo aumento do $\mathrm{pH}$ da solução do solo. Devido a isso, a alcalinidade das águas de irrigação tem que ser reduzida. Para Babcock \& Egorov (1973), a acumulação de carbonato, principalmente de cálcio, pela água de irrigação, pode provocar o processo de cimentação no solo, que pode ocorrer depois de um período de 5-7 anos de irrigação, dificultando assim, a penetração da água de irrigação e de raízes. Em ambiente que possua carbonato de sódio e gesso, pode ocorrer a formação de $\mathrm{Na}_{2} \mathrm{SO}_{4}$ que é muito tóxico para as plantas.

Costa (2000), trabalhando com resposta do arroz à adubação fosfatada associada à correção do solo com calcário em um Latossolo Vermelho-Amarelo Álico em condição de casa-devegetação, observou que, na ausência da calagem o pH do solo, que originalmente era de 5,2, passou para aproximadamente 7,8 , com aplicação diária de água com teor de carbonato e bicarbonato de 0,6 e 2,1 $\mathrm{mmol}_{\mathrm{c}} \mathrm{L}^{-1}$, respectivamente. $\mathrm{O}$ aumento do $\mathrm{pH}$ do solo provocado pela água de irrigação também contribui para a perda de nitrogênio por volatilização, devido o amônio passar para amônia e se perder na forma de gás para a atmosfera em condições alcalina.

Além da aplicação de ácido para baixar o pH da água (Egreja Filho et. al., 1999; Ayers \& Westcot, 1991; Burt et al., 1995), outra alternativa usada atualmente para reduzir o $\mathrm{pH}$ das águas é a injeção de $\mathrm{CO}_{2}$ via água de irrigação. Segundo Storlie \& Heckman (1996) o dióxido de carbono reduz o pH da água e o pH do solo. Essa redução do $\mathrm{pH}$ do solo e da água para níveis ótimos, aumenta a absorção de alguns nutrientes, induzindo a um aumento de produtividade com o uso do $\mathrm{CO}_{2}$ nas águas alcalinas. Basile et al. (1993) mostraram que a disponibilidade de $\mathrm{Zn}, \mathrm{Mn}$, Fe e Ca aumentou com a aplicação de $\mathrm{CO}_{2}$ via água na irrigação localizada. Esse aumento na disponibilidade destes nutrientes foi atribuído ao $\mathrm{pH}$ do solo ter diminuído de 7,5 para 6,0. Bialczyk et al. (1994) também verificaram efeito do $\mathrm{CO}_{2}$ no aumento da produtividade de tomate. Verificaram um aumento na absorção de $\mathrm{N}, \mathrm{K}$ e Ca em plantas cultivadas em solução com diferentes concentrações de $\mathrm{HCO}_{3}{ }^{-}$e concluíram que o efeito positivo do uso do $\mathrm{CO}_{2}$ via água de irrigação só ocorreu em um intervalo limitado de concentração de $\mathrm{HCO}_{3}^{-}$na água.

\section{CONCLUSÕES}

1. As águas da região da Chapada do Apodi apresentaram valores de $\mathrm{E}_{\mathrm{CaCO}_{3}}$ superiores aos da região do Baixo Açu.

2. Independente da origem das águas, para a Chapada do Apodi, a região de Mossoró apresentou os maiores valores de $\mathrm{E}_{\mathrm{CaCO}_{3}}$ com média de $765 \mathrm{~kg} \mathrm{ha}^{-1}$ e os menores valores foram para as regiões de Grossos e Upanema com média de $626 \mathrm{~kg} \mathrm{ha}^{-1}$.

3. Para a região do Baixo Assu, independente da origem, a região de Ipanguassu apresentou os maiores valores de $\mathrm{E}_{\mathrm{CaCO}_{3}}$ com média de $654 \mathrm{~kg} \mathrm{ha}^{-1}$, e os menores valores foram, em média, para a região de Carnaubais com $580 \mathrm{~kg} \mathrm{ha}^{-1}$.

\section{LITERATURA CITADA}

Ayers, R.S.; Westcot, D.W. A qualidade da água na agricultura. Campina Grande, UFPB, 1991. 218p. Estudos FAO: Irrigação e Drenagem, 29

Babcock, K.L.; Egorov, V.V. Chemistry of saline and alkali soil of arid zone. In: Irrigation, drainage and salinity: An international source book. Paris: FAO/UNESCO. 1973. p.122-127.

Basile, G.M.; Arienzo, M.; Zena. A. Soil nutrient mobility in response to irrigation with carbon dioxide enriched water. Communications in Soil Science and Plant Analysis, New York, v.24, 1183-1195, 1993.

Bialczyk, J.; Lechowski, Z.; Libik, A. Growth of tomato seedlings under different $\mathrm{HCO}_{3}{ }^{-}$concentrations in the medium. Journal Plant Nutrition, New York, v.17, n.5, 801-816, 1994.

Burt, C.; O'Connor, K.; Ruehr, T. Fertigation. San Luis Obispo: Carlifórnia Polytechnic State University, Irrigation Training and Research Center, 1995. 295p.

Carmo Filho, F.; Espínola Sobrinho, J.; Maia Neto, J. M. Dados meteorológicos de Mossoró (Jan. de 1988 à Dez. de 1990), Mossoró: ESAM/FGD, 1991. 121p. Coleção Mossoroense 
Costa, S.A.D. Resposta de arroz à adubação fosfatada associada à correção do solo com calcário e metassilicato de cálcio em Latossolo Vermelho-Amarelo álico sob condições de casa de vegetação. Mossoró, RN: ESAM, 2000, 20p. Monografia de Graduação

Egreja Filho, F.B.; Maia, C.E.; Morais, E.R.C. de. Método computacional para correção da alcalinidade de águas para fertirrigação. Revista Brasileira de Ciência do Solo, Viçosa, v.23, n.2, p.415-423, 1999.

Inoue, $\mathrm{A}$. Thermodynamic study of $\mathrm{Na}, \mathrm{K}, \mathrm{Ca}$ exchange reation in vermiculite. Clays \& Mineralogy, v.30, n.4, p.311-319. 1984.

Kelley, R.T. Use of saline irrigation water. Soil Science, Baltimor, v.95, n.6, p.386-391, 1963.

Maia, C.E.; Morais, E.R.C. de; SCASA: Sistema de cadastramento de análises de solo e água. In: Congresso Latino Americano de Ciência do Solo, 13, 1996, Águas de Lindóia. Resumos... Campinas: SBCS, 1996. CD ROM.
Maia, C.E.; Morais, E.R.C. Qualidade da água para fertirrigação por gotejamento. II. Região do Baixo Assu, RN. Engenharia na Agricultura, Viçosa, MG: v.6, n.1, p.12-26. 1998.

Richards, L. A. Diagnosis and improvement of saline and alkali soils. 1.ed. Washigton: US Department of Agriculture, 1954. 160p. USDA Handbook 60

Shainberg, I.; Letey, J. Response of soil to sodic and saline conditions. Hilgardia, Oakland. v.52, n.1, p.1-57. 1984.

Storlie, C.A.; Heckman, J.R. Soil, plant, and canopy responses to carbonated irrigation water. Hort Technology, v.6, n.2, p.111-114, 1996.

Whipker, B.E.; Bailey, D.; Nelson, P.V.; Fonteno, W.C.; Hammer, P.A. A novel approach to calculate acid additions for alkalinity control in greenhouse irrigation water. Communications in Soil Science and Plant Analysis, New York, v.27, p.959-976. 1996. 